\title{
THE APPORTIONMENT OF REPRESENTATIVES IN CONGRESS*
}

\author{
BY \\ E. V. HUNTINGTON
}

\section{INTRODUCTION}

In the absence of any provision for fractional representation in Congress, the constitutional requirement that the number of representatives of each state shall be proportional to the population of that state cannot be carried out exactly; some deviation from strict proportionality is unavoidable, on account of the necessary adjustment of fractions.

Thus, between any two states, there will practically always be a certain inequality which gives one of the states a slight advantage over the other. A transfer of one representative from the more favored state to the less favored state will ordinarily reverse the sign of this inequality, so that the more favored state now becomes the less favored, and vice versa. Whether such a transfer should be made or not depends on whether the amount of inequality between the two states after the transfer is less or greater than it was before; if the "amount of inequality" is reduced by the transfer, it is obvious that the transfer should be made.

The fundamental question therefore at once presents itself, as to how the "amount of inequality" between two states is to be measured. This is a mathematical question of quite unexpected complexity, which has been discussed on a scientific basis only within the last few years. The best solution of the problem appears to be the Method of Equal Proportions, which it is the purpose of the present paper to explain. $\dagger$

* Presented to the Society, December 28, 1920, February 26, April 23, September 8, and December 28, 1921, and February 25, 1922; with the subsequent addition of a number of new examples and tables; and read, in part, before the Mathematical Association of America, December 31, 1926; received by the editors in January, 1927.

† See E. V. Huntington, $A$ new method of apportionment of representatives, Quarterly Publication of the American Statistical Association, September, 1921, pp. 859-870; also the Report upon the Apportionment of Representatives, prepared by the Joint Committee of the American Statistical Association and the American Economic Association to Advise the Director of the Census, and published in the same journal, December, 1921, pp. 1004-1013. This Report, which pronounces in favor of the Method of Equal Proportions, is reprinted in full in Hon. E. W. Gibson's Remarks in the Congressional Record for April 7, 1926, pp. 6840-6842. The Method of Equal Proportions was incorporated in the Bill (H.R. 17378) introduced by Mr. Fenn in the House of Representatives, March 2, 1927; see the Report of Hearings held in January and February, 1927, before the House Committee on the Census (69th Congress, 2d Session), and the Congressional Record for March 2, 1927, pp. 5323-5331. 


\section{A first basis for the Method of EQual Proportions}

The first measure of the "amount of inequality" between two states, which suggests itself, is based on the size of the "congressional district," that is, the result of dividing the population of the state by the number of its representatives.

For example, if the population of a certain state $\mathrm{A}$ is $A=1,000,000$, and the number of its representatives is $a=4$, then the size of a congressional district in that state will be $A / a=250,000$. If the population of a second state $\mathrm{B}$ is $B$ and the number of its representatives is $b$, then the size of the congressional district in the second state is $B / b$.

Now in a perfect apportionment, these two numbers would be exactly equal:

$$
A / a=B / b
$$

hence, in any practical case, the inequality between these two numbersthat is, the inequality between the two congressional districts, $A / a$ and $B / b-$ may be taken as a measure of the "amount of inequality" between the two states $\mathrm{A}$ and $\mathrm{B}$. If this inequality can be reduced by a transfer of a representative from one state to the other, then, according to this first criterion, the transfer should be made.

The rather vague concept of the inequality between two states is thus reduced to the more definite concept of the inequality between two numbers.

The question then comes down to this: what shall be meant by the inequality between these two numbers? Shall we mean the absolute difference between the two numbers, or the relative difference between them? If the size of the congressional districts is large, say 250,000 in one state and 250,005 in the other, then the difference of five people is of little consequence in so large a number. But if the districts were themselves very small, say 10 and 15, then the same difference of five people becomes important; 15, we say, is larger than 10 by fifty per cent, while 250,005 is larger than 250,000 by only (1/500)th of 1 per cent.

In the present problem it is clearly the relative or percentage difference, rather than the mere absolute difference, which is significant.* Our first criterion for a good apportionment may therefore be precisely formulated as follows:

\footnotetext{
* The relative or percentage difference between two numbers is here thought of as the absolute difference divided by the smaller number. For the present purpose it might equally well be thought of as the absolute difference divided by the larger number, or the absolute difference divided by the (arithmetic, geometric, or harmonic) mean between the two numbers.
} 
TEST 1. If the relative difference between the congressional districts, $A / a$ and $B / b$, belonging to any two states can be reduced by a transfer of a representative from one state to the other, then this transfer should be made.

One further question remains. It is not obvious that a transfer which improves the situation between one pair of states, A and B, may not make the situation worse between one of these states and some other state; in other words, it is not obvious that the test can be applied to all pairs of states simultaneously.

It will be shown below, however, that this is a "workable" test; that is, by successive applications of the test, it is always possible to arrive at a final apportionment which cannot be "improved" by any further transfer between any two states.

The only known method of apportionment which satisfies Test 1 proves to be the Method of Equal Proportions.

\section{A second basis for the Method of Equal Proportions}

A second, and equally obvious, method of defining the "amount of inequality" between two states is based, not on the ratio $A / a$, but on the ratio $a / A$ (that is, the number of representatives divided by the population of the state). This number $a / A$ is a small fraction which can be interpreted as the individual share of a representative which each inhabitant in the given state may be said to control.

For example, if the number of inhabitants in a given state is $A=1,000,000$, and the number of representatives is $a=4$, then the "individual share" of a representative which each inhabitant of that state can claim is $a / A$ $=1 / 250,000=0.000004$. If the population of a second state is $B$ and the number of its representatives is $b$, then the "individual share" in the second state is $b / B$.

Now in a perfect apportionment, these two numbers would be exactly equal:

$$
a / A=b / B
$$

hence, in any practical case, the inequality between these two numbersthat is, the inequality between the individual shares, $a / A$ and $b / B$-may be taken as the measure of the "amount of inequality" between the two states $\mathrm{A}$ and $\mathrm{B}$; and here, as before, it is clearly the relative or percentage difference, rather than the mere absolute difference, which is significant.

Our second criterion for a good apportionment may therefore be precisely formulated as follows: 
TEsT 2. If the relative difference between the two "individual shares," a/A and $b / B$, belonging to any two states, can be reduced by a transfer of a representative from one state to the other, then this transfer should be made.

Here again it will be shown that this is a "workable" test; and the only known method of apportionment which satisfies this Test 2 is the same Method of Equal Proportions which also satisfies Test 1.

\section{Working RULe for the Method of Equal Proportions}

We now turn to a purely technical question, of little interest except to the computers in the Bureau of the Census.

Given, the populations of the several states; and given, the size of the House, that is, the total number of representatives to be assigned; how shall we actually compute an apportionment which will satisfy Test 1 and Test 2? The practical working rule for the computation is as follows:

First, assign one representative to each state (here 48 in number).

Next, for each state, make out a series of cards, each card containing: (1) the name of the state; (2) a serial number, $k$, starting with 2 and running up to a number somewhat greater than the number of representatives that that state is expected to receive; and (3) a "rank index," found by multiplying the population of the state by a certain "multiplier," given, for each serial number, in the adjoining table.

Then combine all these series of cards into a single series, arranged in order of the "rank indices," from the highest to the lowest, thus forming what may be called a "priority list," for the given populations, and any size of House.*

\begin{tabular}{|c|c|}
\hline \multicolumn{2}{|c|}{ Method EP } \\
\hline No. & Multiplier \\
\hline 2 & $1 /[(1 \cdot 2)]^{1 / 2}$ \\
3 & $1 /[(2 \cdot 3)]^{1 / 2}$ \\
4 & $1 /[(3 \cdot 4)]^{1 / 2}$ \\
.. & $\cdots \cdots \cdots \cdots$ \\
\hline
\end{tabular}

Finally, assign additional representatives (after the first) to the several states in the order in which the cards occur in this "priority list," continuing the assignment as far as may be necessary to fill up a House of any desired size.

An apportionment worked out according to this rule will always satisfy Test 1 and Test 2, as will be shown below. In practice, it may be found convenient to number the cards of the "priority list" consecutively, in red ink, beginning with the number (here 49) one greater than the number of states, and continuing until any desired total number of representatives

* In case two cards bear the same index number, the state having the larger population may be given priority. This case of a "tie" will be extremely rare, however, on account of the irrationality of the "multipliers" (see a later paragraph). 
(say 435) has been reached. For most purposes, however, the earlier part of the list may be omitted.

In the following table the multipliers are given to seven decimal places.

Table of Multipliers (Method EP)

\begin{tabular}{|c|c|c|c|c|c|c|c|}
\hline No. & Multiplier & No. & Multiplier & No. & Multiplier & No. & Multiplier \\
\hline 1 & & 14 & .0741249 & 27 & .0377426 & 40 & $.0253185-$ \\
\hline 2 & .7071068 & 15 & .0690066 & 28 & .0363696 & 41 & .0246932 \\
\hline 3 & .4082483 & 16 & .0645497 & 29 & .0350931 & 42 & .0240981 \\
\hline 4 & .2886751 & 17 & .0606339 & 30 & .0339032 & 43 & .0235310 \\
\hline 5 & .2236068 & 18 & .0571662 & 31 & .0327913 & 44 & .0229900 \\
\hline 6 & .1825742 & 19 & .0540738 & 32 & $.0317500+$ & 45 & .0224733 \\
\hline 7 & .1543033 & 20 & .0512989 & 33 & .0307729 & 46 & .0219793 \\
\hline 8 & .1336306 & 21 & $.0487950+$ & 34 & .0298541 & 47 & .0215066 \\
\hline 9 & .1178511 & 22 & .0465242 & 35 & .0289886 & 48 & .0210538 \\
\hline 10 & .1054093 & 23 & .0444554 & 36 & .0281718 & 49 & .0206197 \\
\hline 11 & .0953463 & 24 & .0425628 & 37 & .0273998 & 50 & .0202031 \\
\hline 12 & .0870388 & 25 & .0408248 & 38 & .0266690 & 51 & .0198030 \\
\hline 13 & .0800641 & 26 & .0392232 & 39 & .0259762 & & \\
\hline
\end{tabular}

In this table, if $k=$ the serial number, the "multiplier" $=1 /[(k-1) k]^{1 / 2}$. The entries in the table may be verified, with a computing machine, by the process of squaring and taking reciprocals, without extracting square roots.

An illustration of the use which may be made of the table of multipliers, even before the priority list is completed, is the following: Any state A will receive its $43 \mathrm{~d}$ representative before another State $B$ receives its 8 th representative, provided the population of State A multiplied by 0.0235310 is greater than the population of State B multiplied by 0.1336306 .

Since the typical multiplier, $1 /[x(x+1)]^{1 / 2}$, is the reciprocal of the geometric mean between the successive integers $x$ and $x+1$, the Method of Equal Proportions might be called also the Method of the Geometric Mean.*

The proof of the correctness of the rule for Method EP is as follows.

Suppose that, in an apportionment made according to the rule, any State A has received $x+1$ representatives and any other State B has received $y$ representatives; and suppose (as we may, without loss of generality) that

- The first use of the geometric mean in connection with this problem occurs in the Method of Alternate Ratios, proposed by Dr. J. A. Hill in 1910; this method, though obtained through entirely different reasoning (Tests 1 and 2 being unknown at that time), differs from the Method of Equal Proportions only in the fact that it insists on too close a relationship between the assignment given to any state and the true quota of that state. (This defect leads to an "Alabama paradox," as we shall see below.) Dr. Hill was also the first writer to recognize the superiority of the relative difference over the absolute difference, in the solution of this problem. See his paper in House of Representatives Report No. 12, of the Sixty-Second Congress, First Session, April 25, 1911. 
State $\mathbf{A}$ is over-represented in comparison with State B. We proceed to show that if one representative is transferred from State $A$ to State $B$, the "inequality" between the two states (measured according to Test 1 or Test 2) will be thereby increased.

We begin by showing that in the hypothetical apportionment, in which State A has $x$ representatives and State B has $y+1$, the latter state will be over-represented in comparison with the former.

From the way in which the "priority list" is constructed we know that $A^{2} /[x(x+1)]>B^{2} /[y(y+1)]$; and since in the actual assignment $\mathrm{A}$ is overrepresented in comparison with $\mathrm{B}$, we know that $B / y>A /(x+1)$, and hence $(x+1) / A>y / B$. It follows that $B /(y+1)<A / x$, and hence $x / A<(y+1) / B$, since the contrary assumption would lead to contradiction. But these last relations express the fact that after the transfer is made, State B is overrepresented in comparison with State A.

We can now write down the expression for the "inequality" between the two states before and after the transfer, remembering the convention that the "percentage difference" between any two numbers is understood to mean the "absolute difference divided by the smaller number."

In the actual assignment (before the transfer), the inequality in question is, by Test $1,[B / y-A /(x+1)] /[A /(x+1)]$, or, by Test $2,[(x+1) / A-y / B]$ $/[y / B]$, each of which reduces to $B(x+1) /(A y)-1$.

In the hypothetical asisignment (after the transfer), the inequality is, by Test $1,[A / x-B /(y+1)] /[B /(y+1)]$, or, by Test $2,[(y+1) / B-x / A]$ $/[x / A]$, each of which reduces to $A(y+1) /(B x)-1$.

But from the given relation $A^{2}[x(x+1)]>B^{2} /[y(y+1)]$, we have at once $A^{2}(y+1) / x>B^{2}(x+1) / y$, whence

$$
A(y+1) /(B x)-1>B(x+1) /(A y)-1,
$$

which shows that the inequality between the two states would be increased by the transfer.

In other words, an apportionment made according to the rule, is one which cannot be "improved" (in the sense of Test 1 or Test 2), by any transfer of a representative from any state to any other state.

\section{CRItique of TWO CONFLiCting MEthodS}

As pointed out above, whichever definition of the amount of inequality between two states may be adopted, it is clearly the relative or percentage difference, rather than the mere absolute difference, which is significant. The inappropriateness of the absolute difference is made still more apparent by the fact that its use leads us to two conflicting methods of apportionment. 
Thus, if we substitute the "absolute difference" for the "relative difference" in Tests 1 and 2,, we have the following tests:

TEST 1a (not recommended). If the absolute difference between the two congressional districts, $A / a$ and $B / b$, can be reduced by a transfer of a representative from one state to the other, then this transfer should be made.

This test leads to a distinct method of apportionment, known as the Method of the Barmonic Mean (HM).*

TEST 2a (not recommended). If the absolute difference between the two "individual shares," $a / A$ and $b / B$, can be reduced by a transfer of $a$ representative from one state to the other, then this transfer should be made (except that no state shall be left without at least one representative).

This test leads to another distinct method of apportionment, known as the Method of Major Fractions (MF). $\dagger$

Thus, while each of these tests is a "workable" test, each leads to a distinct method of apportionment. In comparison with the Method of Equal Proportions, the Method of the Harmonic Mean favors the small states unduly, while the Method of Major Fractions favors the large states unduly. This is illustrated in Example 1, showing the apportionment of 16 representatives among three states with a total population of 1600 .

In this example, it is fairly obvious that State A should have at least 7 representatives, State $B$ at least 5 , and State $C$ at least 3. But this makes only 15 in all. Where shall the 16th representative be assigned? Method HM gives it to the smallest state (C), and Method MF gives it to the largest state (A); while the Method of Equal Proportions gives it to the middlesized state (B).

The computations in the right-hand part of the table will be selfexplanatory. Method HM differs from Method EP only in regard to States $\mathrm{B}$ and C; the "amount of inequality" between these two states is smaller in

\footnotetext{
- See E. V. Huntington, in the paper already cited; or a brief abstract entitled The mathematical theory of the apportionment of representatives, in the Proceedings of the National Academy of Sciences, vol. 7, pp. 123-127, April, 1921.

t The Method of Major Fractions was devised by Professor W. F. Willcox in 1910, and was used in the apportionment for that year. See his paper in House of Representatives Report No. 12, of the Sixty-Second Congress, First Session, April 25, 1911, and his presidential address as president of the American Economic Association, published in the American Economic Review, vol. 6, no. 1, Supplement, pp. 1-16, March, 1916; also F. W. Owens, On the apportionment of representatives, Quarterly Publication of the American Statistical Association, December, 1921, pp. 958-968. The Report of the Advisory Committee cited above, concluded, after elaborate hearings, that the Method of Major Fractions was less desirable than the Method of Equal Proportions.
} 


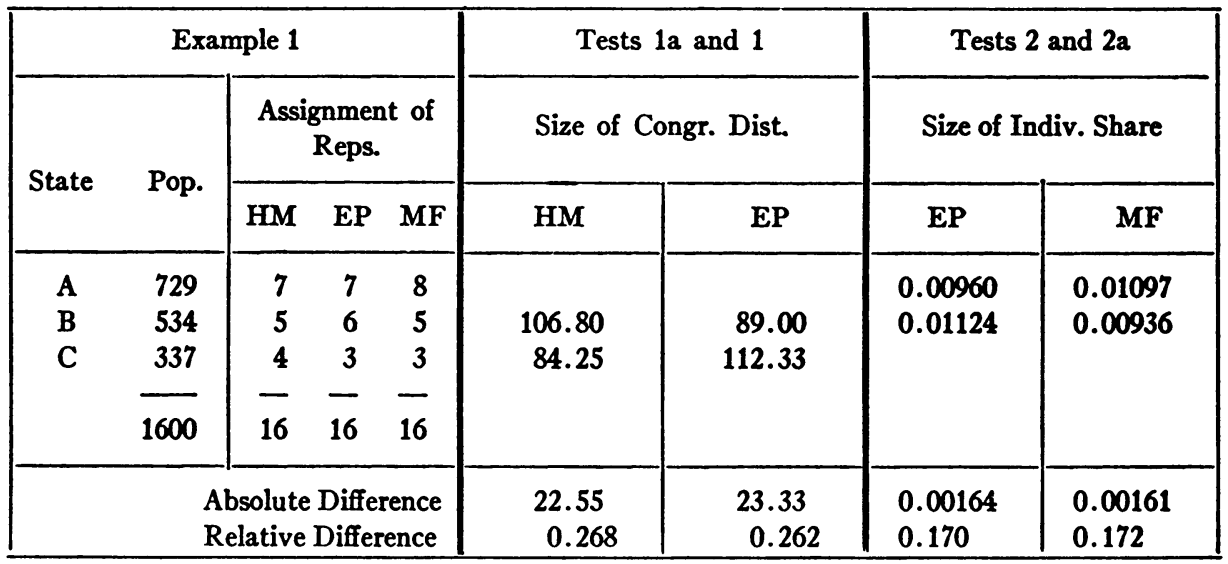

column HM when Test 1a is used, and smaller in column EP when Test 1 is used. Similarly, Method MF differs from Method EP only in regard to States A and B; the "amount of inequality" between these two states is smaller in column MF when Test 2a is used, and smaller in column EP when Test 2 is used.

Thus Tests 1a and 2a lead to conflicting results (Methods HM and MF); if these were the only tests available, it would be difficult to make a choice between them on any but arbitrary grounds.

On the other hand, Tests 1 and 2 lead to no such dilemma, since the Method of Equal Proportions satisfies them both. This fact strengthens our belief that in defining a measure of inequality between two states, the relative difference is more natural and useful than the absolute difference.* The two conflicting Methods, HM and MF, may be regarded as on the same level of merit, as between themselves; but both of them are inferior to the Method of Equal Proportions.

\section{WORKING RULE FOR METHOD OF HARMONIC MEAN}

The working rule for the Method of the Harmonic Mean is the same as the rule for the Method of Equal Proportions, if, in forming the "priority list," we replace the series of multipliers, there given, by the following:

$$
\frac{1}{2(1 \cdot 2) /(1+2)}, \quad \frac{1}{2(2 \cdot 3) /(2+3)}, \quad \frac{1}{2(3 \cdot 4) /(3+4)}, \cdots
$$

\footnotetext{
- See, however, Test 33, below, which uses either the absolute or the relative difference at pleasure.
} 
The name Method of the Harmonic Mean is suggested by the fact that the typical multiplier, $1 /[2(x)(x+1) /(x+x+1)]$, is the reciprocal of the harmonic mean between the successive integers $x$ and $x+1$.

The proof that this rule will result in an apportionment satisfying Test 1a is as follows: Suppose, as before, that State $A$ has $x+1$ representatives, and State $B$ has $y$ representatives, and that State $A$ is over-represented in comparison with State B. Then the inequality between these two states, measured according to Test $1 \mathrm{a}$, is $B / y-A /(x+1)$. If, on the other hand, State A had only $x$ representatives and State $B$ had $y+1$, then the inequality between the two states would be $A / x-B /(y+1)$. From the way in which the priority list is constructed, we know that $A(x+x+1) /[2(x)(x+1)]$ $>B(y+y+1) /[2(y)(y+1)]$, whence $A / x+A /(x+1)>B / y+B /(y+1)$, whence $A / x-B /(y+1)>B / y-A /(x+1)$.

\section{WORKING RULE FOR THE METHOd OF MAJOR Fractions}

The working rule for the Method of Major Fractions is the same as the rule for the Method of Equal Proportions, with the replacement of the series of multipliers, there given, by the following:

$$
\frac{1}{1+\frac{1}{2}}, \quad \frac{1}{2+\frac{1}{2}}, \quad \frac{1}{3+\frac{1}{2}}, \cdots
$$

Since the typical multiplier, $1 /\left(x+\frac{1}{2}\right)$, is the reciprocal of the arithmeti mean between the successive integers $x$ and $x+1$, the method might bc called the Method of the Arithmetic Mean. The name "Method of Major Fractions," which is now well established, is due to Professor W. F. Willcox, who, approaching the subject from an entirely different point of view, devised the working rule, as a practical method of computation, in 1910, at a time when none of the theoretical tests $(1,2,1 a, 2 a)$ were known. The Method of Major Fractions satisfies none of these tests except Test 2a.

The proof that this rule will result in an apportionment satisfying Test 2a is as follows: Suppose, as before, that State A has $x+1$ representatives, and State B has $y$ representatives, and that State A is over-represented in comparison with State B. Then the inequality between those two states, measured by Test 2a, is $(x+1) / A-y / B$. If, on the other hand, State $A$ had $x$ and State $B$ had $y+1$, then the inequality between the two states would be $(y+1) / B-x / A$. Now, from the way in which the priority list is constructed, $A /\left(x+\frac{1}{2}\right)$ is greater than $B /\left(y+\frac{1}{2}\right)$; hence $(2 y+1) / B>(2 x+1) / A$, whence $(y+1) / B-x / A>(x+1) / A-y / B$, which was to be proved. 
Remarks on the NAME "Method of Major Fractions" AND THE "EXACT QUOTA" OF A STATE

As a question of practical politics, the controversy at the present time is chiefly between the Method of Equal Proportions (EP) and the Method of Major Fractions (MF).

To avoid any possible misinterpretation of the name "Method of Major Fractions," the following remarks are here inserted.

In a theoretically perfect apportionment, the exact quota of any state $\mathbf{A}$ is $A(R / P)$, where $A$ is the population of the state, $R$ is the total number of representatives in the House, and $P$ is the total population of the country. If the exact quotas of all the states came out as whole numbers, the problem of apportionment would be solved without further ado. But in practically all cases, the exact quota will not be a whole number, and the actual assignment must be greater or less than the quota.

Now it is a common misconception that in a good apportionment the actual assignment should not differ from the exact quota by more than one whole unit; for example, if the exact quota is 5.21 or 5.76 , then it is often assumed that the actual assignment should not be less than 5 nor more than 6 .

It is a further misconception that if the exact quota is, say, 5 and a fraction, then if the fraction is less than $1 / 2$ it should be disregarded, but if it is greater than $1 / 2$, it should add one to the assignment. For example, it is often assumed that if the quota is 5.21, the assignment should be 5; and if the quota is 5.76, the assignment should be 6 .

As a matter of fact, however, neither of these principles is a workable test of a good apportionment, and the Method of Major Fractions, like every other known method of apportionment, will often violate both of them.

Thus, in Example 2, both the Method of Major Fractions and the Method EP assign only 90 representatives to State A, although the exact quota of that state is 92.15 .

Again, in Example 3, both methods assign 90 representatives to State A, although the exact quota of that state is only 87.85 .

Further, in Example 4, the true quota of State $\mathrm{A}$ is 9.87 ; but both methods give State A only 9 representatives, in spite of the fact that the fraction 0.87 is very much greater than $1 / 2$, and is, in fact, the largest of the three fractions which occur in this example.

Again, in Example 5, the true quota of State A is 7.31; but both methods give this state 8 representatives, in spite of the fact that the fraction 0.31 
is less than $1 / 2$, and is, in fact, the smallest of the three fractions that occur in this example.

Although crucial examples of this sort are not easy to construct, the existence of these examples is sufficient to show that the "Method of Major Fractions" does not imply that a "major fraction" in the quota of a state will always entitle that state to an additional representative, or that a "minor fraction" is always to be disregarded.

As a matter of fact, the size of the quota of an individual state, taken by itself, does not determine the number of representatives to which that state is entitled. For instance, in Examples 5 and 5a, the quota of State B is the same in both cases (5.35); and yet (according to either Method MF or Method EP), the number of representatives assigned to this state is 5 in one case and 6 in the other. This variation in the assignment given to State B is due not to any change in State B itself, but to a slight shift of population between the other two states.

Example 2

\begin{tabular}{|lr|r|}
\hline State & Pop. & EP \\
MF \\
\cline { 1 - 2 } A & 9215 & 90 \\
B & 159 & 2 \\
C & 158 & 2 \\
D & 157 & 2 \\
E & 156 & 2 \\
F & 155 & 2 \\
& $\underline{10,000}$ & $\underline{100}$ \\
\hline
\end{tabular}

Example 3

\begin{tabular}{|lr|r|}
\hline State & Pop. & $\begin{array}{l}\text { EP } \\
\text { MF }\end{array}$ \\
\hline A & 8785 & 90 \\
B & 126 & 1 \\
C & 125 & 1 \\
D & 124 & 1 \\
E & 123 & 1 \\
F & 122 & 1 \\
G & 121 & 1 \\
H & 120 & 1 \\
I & 119 & 1 \\
J & 118 & 1 \\
K & 117 & 1 \\
& 10,000 & 100 \\
\hline
\end{tabular}

Example 4

\begin{tabular}{|cc|c|}
\hline State & Pop. & EP \\
\hline AF \\
B & 987 & 9 \\
C & 157 & 2 \\
& 156 & 2 \\
& 1300 & 13 \\
\hline
\end{tabular}

Example 5

\begin{tabular}{|lc|c|}
\hline State & Pop. & $\begin{array}{c}\text { EP } \\
\text { MF }\end{array}$ \\
\hline A & 731 & 8 \\
B & 535 & 5 \\
C & 334 & 3 \\
& 1600 & - \\
\hline
\end{tabular}

Example 5a

\begin{tabular}{|cc|c|}
\hline State & Pop. & EP \\
MF
\end{tabular}


Again, in Examples 6 and 6a, the quota for State B is exactly 44 in each case; but the actual assignment (according to either Method MF or Method $\mathrm{EP}$ ) is 43 in one case and 45 in the other.

Example 6

\begin{tabular}{|cr|r|r|}
\hline State & Pop. & Quota & \multicolumn{1}{|c|}{$\begin{array}{c}\text { EP } \\
\text { MF }\end{array}$} \\
\hline A & 5117 & 51.17 & 51 \\
B & 4400 & 44.00 & 43 \\
C & 162 & 1.62 & 2 \\
D & 161 & 1.61 & 2 \\
E & 160 & 1.60 & 2 \\
& & & \\
& 10,000 & 100 & 100 \\
\hline
\end{tabular}

Example 6a

\begin{tabular}{|c|c|c|c|}
\hline State & Pop. & Quota & $\begin{array}{r}\text { EP } \\
\text { MF }\end{array}$ \\
\hline $\mathbf{A}$ & 5189 & 51.89 & 52 \\
\hline B & 4400 & 44.00 & 45 \\
\hline C & 138 & 1.38 & 1 \\
\hline D & 137 & 1.37 & 1 \\
\hline \multirow[t]{2}{*}{$\mathbf{E}$} & 136 & 1.36 & 1 \\
\hline & 10,000 & 100 & 100 \\
\hline
\end{tabular}

Example 7

\begin{tabular}{|cr|r|r|}
\hline State & Pop. & Quota & $\begin{array}{r}\text { No. of } \\
\text { Reps. }\end{array}$ \\
\hline A & 1536 & 15.36 & 15 \\
B & 1535 & 15.35 & 15 \\
D & 1534 & 15.34 & 15 \\
E & 1533 & 15.33 & 15 \\
F & 1532 & 15.32 & 15 \\
G & 1530 & 15.30 & 15 \\
H & 162 & 1.62 & 2 \\
I & 161 & 1.61 & 2 \\
J & 160 & 1.60 & 2 \\
K & 158 & 1.59 & 2 \\
& 10,000 & 100 & 2 \\
\hline Group & Pop. & Quota & Reps. \\
\hline ABCDEF & 9200 & 92.00 & 90 \\
GHIJK & 800 & 8.00 & 10 \\
& 10,000 & 100 & 100 \\
\hline \hline
\end{tabular}

Furthermore Example 7 shows that "nearness to the quota" with respect to groups of states is incompatible with "nearness to the quota" with respect to single states.

In this example, the quota of the group of large states is 92, while the actual assignment to this group is only 90; and the quota for the group of small states is 8 , while the actual assignment to this group is 10 . A transfer of a representative from the small group to the large group (say from State $\mathbf{K}$ to State A) would bring both groups "nearer to the quota"; and yet no one would wish to make this transfer.

In short, "nearness to the quota" cannot be taken as a test of a good assignment, either for a single state or for a group of states.

\section{REMARKS ON THE WILLCOX SLIDING DIVISOR}

The origin of the name "Method of Major Fractions" is to be found in an ingenious device known as the "sliding divisor," and due, in its present form, to Professor Willcox. 
After an apportionment has been computed by the working rule for the Method of Major Fractions, the sliding devisor may be used to facilitate the recording of the results. This device is supplementary to the actual computation and forms no essential part of it; it is interesting chiefly as explaining the origin of the name.

The device consists in the selection of any number $W$ such that

$$
\frac{A}{a-\frac{1}{2}}>W>\frac{B}{a+\frac{1}{2}}, \quad \frac{B}{b-\frac{1}{2}}>W>\frac{B}{b+\frac{1}{2}}, \quad \frac{C}{c-\frac{1}{2}}>W>\frac{C}{c+\frac{1}{2}} \text {, etc. , }
$$

where $a, b, c$, etc., are the assignments of representatives to the States A, B, C, etc., according to Method MF.

Such a number $W$, which may be called a Willcox Divisor, will always exist, ${ }^{*}$ and will have the following property: If the population of each State is divided by $W$, there will be obtained a series of quotients such that, if one representative is assigned for each unit and for each major fraction (and also for each quotient which is itself less than one-half), the resulting apportionment will be precisely the same as the apportionment given by the working rule for the Method MF, and will therefore satisfy Test $2 \mathrm{a}$.

By the use of this device, the assignment given to any State can be figured out at once from the population of that State, as soon as the value of the Willcox divisor has been announced.

It should be noticed, however, that the Willcox divisor is not the true value of the average Congressional District, and the Willcox quotients are not the true quotas of the several states; hence the occurrence of a major fraction in the "quotient" of a State gives that State no claim whatever to an additional representative, except the claim which is already implied by Test $2 a$. If a method could be found which would assign an additional representative for every major fraction in the true quota, it would be indeed a simple and attractive method; but as we have seen, no such method is possible.

The Willcox "sliding divisor" merely provides a convenient way of recording the result of the method based on Test $2 a$, and adds nothing (except the name) to the authority of that method. The simplest basis for a valid method is a direct comparison between competing States, as expressed in Test 1 or Test 2; and the only method which satisfies either of these simple and natural tests is the Method of Equal Proportions.

\section{Note on the Alabama paradox}

The curious situation known as the "Alabama Paradox" is a further illustration of the confusion resulting from the unwise use of the exact quota of a state in computing the apportionment.

\footnotetext{
- Except in the case of a "tie" between two states (see below).
} 
This paradox first came to the attention of Congress in the tables prepared in 1881, which gave Alabama 8 members in a House of 299, and only 7 members in a House of 300, so that an increase in the total size of the House actually produced a decrease in the number of representatives of one of the states.

The method in use at that time was known as the Vinton Method of 1850. This method assumed that each state was entitled to at least as many representatives as was indicated by the largest whole number contained in the exact quota of that state (with the special provision that no state should have less than one representative). To fill up the required total, further representatives were then assigned, "for fractions," to as many states as necessary, the states being arranged, for this latter purpose, in a "priority list," according to the magnitude of the fractions themselves, so that the state with the largest fraction was the first to receive an additional representative.

The resulting paradox is illustrated in Example 8, where State C has 11 representatives in a House of 100 members, and only 10 representatives in a House of 101.

A similar defect occurs in the otherwise excellent Method of Alternate Ratios proposed by Dr. J. A. Hill in 1910. This method proceeds as in the Vinton Method, except that the "priority list" for fractions is arranged according to the magnitude of the quantity $A /[x(x+1)]^{1 / 2}$, where $x$ is the number already assigned to State $\mathrm{A}$, and $x+1$ is the next larger number. The possible paradox resulting from this method is shown in Example 8a, where States $\mathrm{G}$ and $\mathrm{H}$ each lose one representative when the size of the House is increased from 100 to 101.

No method can be regarded as satisfactory which is subject to the Alabama Paradox.

Example 8

Vinton Method (Paradox)

\begin{tabular}{|rr|r|r|r|r|}
\hline \multirow{2}{*}{ Pop. } & \multicolumn{2}{|c|}{100} & \multicolumn{2}{c|}{101} \\
\cline { 2 - 6 } & & Quota & Rep. & Quota & Rep. \\
\hline A & 453 & 45.3 & 45 & 45.753 & 46 \\
B & 442 & 44.2 & 44 & 44.642 & 45 \\
C & 105 & 10.5 & 11 & 10.605 & 10 \\
& $\frac{10}{1000}$ & 100.0 & 100 & 101.000 & $\frac{101}{101}$ \\
\hline
\end{tabular}

Example 8a Method of Alternate Ratios (Paradox)

\begin{tabular}{|rr|r|r|r|r|}
\hline & & \multicolumn{2}{|c|}{100} & \multicolumn{2}{|c|}{101} \\
\cline { 2 - 6 } & Pop. & Quota & Rep. & Quota & Rep. \\
\hline A & 154550 & 30.91 & 30 & 31.2191 & 31 \\
B & 154500 & 30.90 & 30 & 31.2090 & 31 \\
C & 154450 & 30.89 & 30 & 31.1989 & 31 \\
D & 7400 & 1.48 & 2 & 1.4948 & 2 \\
E & 7350 & 1.47 & 2 & 1.4847 & 2 \\
F & 7300 & 1.46 & 2 & 1.4746 & 2 \\
G & 7250 & 1.45 & 2 & 1.4645 & 1 \\
H & 7200 & 1.44 & 2 & 1.4544 & 1 \\
& \multicolumn{1}{|c|}{} & & & & \\
\hline & 500000 & 100.00 & 100 & 101.0000 & 101 \\
\hline
\end{tabular}


Note ON THE CASE OF A TIE BETWEen TWO STATES

In applying the rule for the Method of Equal Proportions, the case of a "tie" between two states can occur only extremely rarely, that is to say, only when two "multipliers" (used in forming the priority list) happen to be commensurable numbers. For serial numbers up to $k=100$, this occurs only four times, as follows:

\begin{tabular}{c|c}
$k$ & $1 /$ mult. \\
\hline 25 & $10(6)^{1 / 2}$ \\
3 & $(6)^{1 / 2}$
\end{tabular}

\begin{tabular}{c|c}
$k$ & $1 /$ mult. \\
\hline 49 & $28(3)^{1 / 2}$ \\
4 & $2(3)^{1 / 2}$
\end{tabular}

\begin{tabular}{c|r}
$k$ & $1 /$ mult. \\
\hline 50 & $35(2)^{1 / 2}$ \\
9 & $6(2)^{1 / 2}$
\end{tabular}

\begin{tabular}{r|r}
\multicolumn{1}{c|}{$k$} & $1 /$ mult. \\
\hline 81 & $\begin{array}{r}36(5)^{1 / 2} \\
5\end{array}$ \\
$2(5)^{1 / 2}$
\end{tabular}

The corresponding "ties" for the Method EP are as follows:

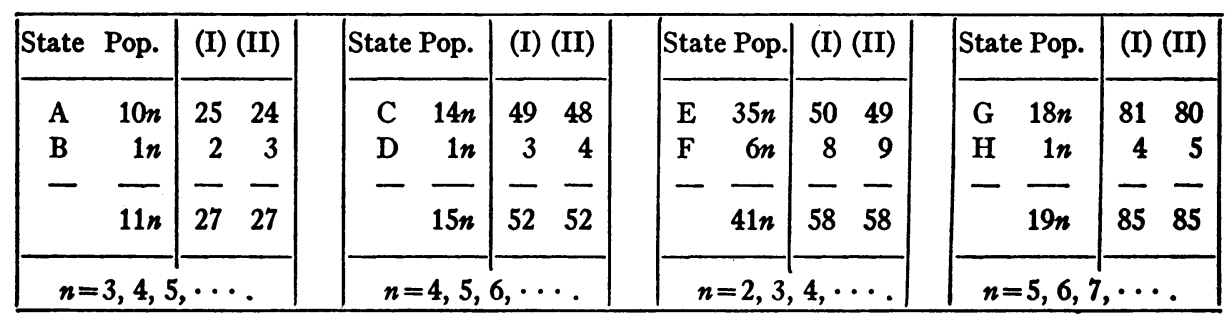

In each of these four cases, assignment (I) is chosen rather than assignment (II) merely on account of the convention which provides that in case of a tie preference shall be given to the state having the larger population.

On the other hand,in applying the rule for the Method of Major Fractions, the case of a tie may occur much more frequently. Thus, if $p, q$, and $n$ are any positive integers, the following assignments (I) and (II) will always be tied in the Method MF:

\begin{tabular}{|c|c|c|c|}
\hline State & Pop. & (I) & (II) \\
\hline \multirow{3}{*}{$\begin{array}{l}\mathbf{J} \\
\mathbf{K}\end{array}$} & $(2 p+1) n$ & $p+1$ & $p$ \\
\hline & $(2 q+1) n$ & $q$ & $q+1$ \\
\hline & $2(p+q+1) n$ & $p+q+1$ & $p+q+1$ \\
\hline
\end{tabular}


We may even have a triple tie, as follows:

\begin{tabular}{|cr|ccc|}
\hline \multirow{2}{*}{ State } & Pop. & \multicolumn{3}{|c|}{ Method MF } \\
\cline { 3 - 5 } & (I) & (II) & (III) \\
\hline L & 11000 & 6 & 5 & 5 \\
M & 7000 & 3 & 4 & 3 \\
N & 3000 & 1 & 1 & 2 \\
& $\frac{1}{21000}$ & $\frac{1}{10}$ & $\frac{1}{10}$ & $\frac{1}{10}$ \\
\hline
\end{tabular}

While none of these tie cases is likely to occur in actual practice in Congress, the extreme rarity of the possibility of such a tie in the Method of Equal Proportions is a theoretical argument in favor of that method.

\section{APPENDIX I}

\section{CritiQue OF TWO FURTHER CONFLICTING METHODS}

A third form in which the exact equality between two states may be written is

$$
\text { (rep. over) }=(\text { rep. under }) \frac{\text { (Pop. over) }}{\text { (Pop. under) }} \text {, }
$$

where "Pop. over" and "rep. over" stand for the population and number of representatives of that one of the two states which is over-represented in comparison with the other, and "Pop. under" and "rep. under" stand for the population and number of representatives of the under-represented state.

The (relative or absolute) difference between the two sides of this equation may be taken as a third measure of inequality between the two states, and may be called the (relative or absolute) "representation-surplus" belonging to the two states. If we use the relative difference, we obtain a third test, which we may call Test 3 (not written out here in detail), which leads to the Method of Equal Proportions. If we use the absolute difference, we have the following less desirable test:

TEst 3a (not recommended). If the absolute "representation-surplus" belonging to any two states, that is, the value of

$$
\text { (rep. over) - (rep. under) }[(\text { Pop. over }) /(\text { Pop. under })] \text {, }
$$

can be reduced by a transfer of a representative from one state to the other, then this transfer should be made.

This Test 3a proves to be a "workable" test, and leads to a distinct method of apportionment which may be called the Method of Smallest 
Divisors (SD). In comparison with the Method of Equal Proportions, Method SD favors the small states even more than does the Method of the Harmonic Mean (see Example 9).

A fourth form of the exact equation, namely,

$$
\text { (rep. over) } \frac{\text { (Pop. under) }}{\text { (Pop. over) }}=\text { (rep. under), }
$$

suggests, in a similar way, a Test 4 , based on relative differences and leading to the Method of Equal Proportions, and a less desirable Test 4a, based on absolute differences, as follows:

TEsT 4a (not recommended). If the absolute "representation-deficiency" belonging to any two states, that is, the value of

(rep. over) $[($ Pop. under $) /($ Pop. over $)]-$ (rep. under), can be reduced by a transfer of a representative from one state to the other, then this transfer should be made.*

This Test 4a proves to be "workable," and leads to another distinct method of apportionment which may be called the Method of Greatest Divisors (GD). In comparison with the Method of Equal Proportions, Method GD favors the large states even more than does the Method of Major Fractions (see Example 9).

\begin{tabular}{|c|c|c|c|c|c|c|c|c|}
\hline \multicolumn{5}{|c|}{ Example 9} & \multirow{2}{*}{\multicolumn{2}{|c|}{$\frac{\text { Tests } 3 \mathrm{a} \text { and } 3}{\text { Representation-Surplus }}$}} & \multirow{2}{*}{\multicolumn{2}{|c|}{$\frac{\text { Tests } 4 \text { and } 4 a}{\text { Representation-Deficiency }}$}} \\
\hline \multirow[b]{3}{*}{ State } & \multirow[b]{3}{*}{ Pop. } & \multicolumn{3}{|c|}{$\begin{array}{l}\text { Assignment of } \\
\text { Reps. }\end{array}$} & & & & \\
\hline & & & HM & & $4-5 C / B$ & $6-3 B / C$ & $6 A / B-7$ & $8 B / A-5$ \\
\hline & & SD & EP & GD & SD & EP & EP & GD \\
\hline $\mathbf{A}$ & 726 & 7 & 7 & 8 & & & 7.000 & 5.939 \\
\hline B & 539 & 5 & 6 & 5 & 3.108 & 6.000 & 8.082 & 5.000 \\
\hline C & 335 & 4 & 3 & 3 & 4.000 & 4.827 & & \\
\hline & $\overline{1600}$ & $\overline{16}$ & $\overline{16}$ & $\overline{16}$ & & & & \\
\hline & & $\begin{array}{l}\text { bsolu } \\
\text { elati }\end{array}$ & & & $\begin{array}{l}0.892 \\
0.287\end{array}$ & $\begin{array}{l}1.173 \\
0.243\end{array}$ & 1.082 & 0.939 \\
\hline
\end{tabular}

The conflict between Tests 3a and 4a, which does not exist between Tests 3 and 4, again confirms our belief that the relative difference is, for the present

- Tests 3a and 4a were presented by the present writer at a meeting of the American Mathematical Society on February 25, 1922. 
problem, a more natural and useful idea than the absolute difference. The two conflicting Methods SD and GD may be regarded as on the same level of merit, as between themselves; but both of them are inferior to Methods HM and MF, and even more inferior to the Method of Equal Proportions.

\section{Working RULE for the Method of SMallest Divisors}

The working rule for Method SD is the same as the rule for Method EP, except that, in forming the "priority list," the multipliers there given are replaced by those in the adjoining table.

The proof that this rule satisfies Test $3 \mathrm{a}$ is as follows. Suppose, as before, that A has $x+1$ and B has $y$, and that State $A$ is over-represented in comparison with State B. Then the inequality between States $A$ and $B$, measured according to Test $3 a$, is $(x+1)-y(A / B)$. If, hypothetically, A had $x$ and B

\begin{tabular}{|c|c|}
\hline \multicolumn{2}{|c|}{ Method SD } \\
\hline No. & Multipliers \\
\hline 2 & $1 / 1$ \\
3 & $1 / 2$ \\
4 & $1 / 3$ \\
- & - \\
\hline
\end{tabular}
had $y+1$, then the inequality would be $(y+1)-x(B / A)$. Now from the construction of the "priority list," $A / x>B / y$; hence

$$
1+(A+B) y / B>1+(A+B) x / A ;
$$

hence

$$
y+1+A y / B>x+1+B x / A,
$$

whence

$$
(y+1)-x(B / A)>(x+1)-y(A / B) .
$$

\section{Working RULE fOR the Method of Greatest Divisors}

The working rule for Method GD is the same as the rule for Method EP, except that the table of "multipliers" is replaced by the table here given.

The proof that this rule satisfies Test $4 \mathrm{a}$ is, briefly, as follows: If $\mathrm{A}$ has $x+1$ and $\mathrm{B}$ has $y$, the inequality, according to Test $4 \mathrm{a}$, is $(x+1) B / A-y$. If $\mathrm{A}$ had $x$ and $\mathrm{B}$ had $y+1$, the inequality would be $(y+1) A / B-x$. Now from the construction of the "priority list," $A /(x+1)>B /(y+1)$; hence

\begin{tabular}{|c|c|}
\hline \multicolumn{2}{|c|}{ Method GD } \\
\hline No. & Multipliers \\
\hline 2. & $1 / 2$ \\
3 & $1 / 3$ \\
4 & $1 / 4$ \\
- & - \\
\hline
\end{tabular}




$$
(A+B)(y+1) / B>(A+B)(x+1) / A,
$$

whence

whence

$$
(y+1) A / B+y+1>(x+1) B / A+x+1,
$$

$$
(y+1) A / B-x>(x+1) B / A-y .^{*}
$$

\section{COMPARISON OF THE FIVE KNOWN METHODS OF APPORTIONMENT}

The only known methods of apportionment which are "workable," and avoid the Alabama Paradox, are the five methods described above, namely (in the order in which they favor the smaller states), Methods SD, HM; EP; MF, GD.

Example 10 (which is a combination of Examples 1 and 9) gives a comparison of the results of all five of the methods. Examples 11 and 12

\begin{tabular}{|c|c|c|c|c|c|c|c|c|c|c|c|c|c|c|c|c|c|c|c|}
\hline \multirow[b]{3}{*}{ Pop. } & \multicolumn{5}{|c|}{ Example 10} & \multicolumn{7}{|c|}{ Example 11} & \multicolumn{7}{|c|}{ Example 12} \\
\hline & \multicolumn{5}{|c|}{ Number of Reps. } & \multirow{2}{*}{\multicolumn{2}{|c|}{ Pop. }} & \multirow{2}{*}{\multicolumn{5}{|c|}{$\frac{\text { Number of Reps. }}{\text { SD HM EP MF GD }}$}} & \multicolumn{7}{|c|}{ Number of Reps. } \\
\hline & \multicolumn{5}{|c|}{ SD HM EP MF GD } & & & & & & & & \multicolumn{2}{|c|}{ Pop. } & \multicolumn{5}{|c|}{ SD HM EP MF GD } \\
\hline 729 & 7 & 7 & 7 & 8 & 8 & & 762 & 7 & 8 & 8 & 8 & 8 & & 9061 & 9 & 9 & 9 & 9 & 10 \\
\hline B 726 & 7 & 7 & 7 & 7 & 8 & B & 758 & 7 & 7 & 7 & 8 & 8 & & 7179 & 7 & 7 & 7 & 8 & 7 \\
\hline C 539 & 5 & 6 & 6 & 6 & 5 & C & 555 & 6 & 5 & 6 & 5 & 6 & & 5259 & 5 & 5 & 6 & 5 & 5 \\
\hline D 534 & 5 & 5 & 6 & 5 & 5 & D & 351 & 4 & 4 & 3 & 3 & 3 & D & 3319 & 3 & 4 & 3 & 3 & 3 \\
\hline E 337 & 4 & 4 & 3 & 3 & 3 & E & 174 & 2 & 2 & 2 & 2 & 1 & $\mathbf{E}$ & 1182 & 2 & 1 & 1 & 1 & 1 \\
\hline F 335 & 4 & 3 & 3 & 3 & 3 & & - & - & - & - & - & - & & & - & - & 一 & 一 & - \\
\hline & - & - & - & - & - & & 2600 & 26 & 26 & 26 & 26 & 26 & & 26000 & 26 & 26 & 26 & 26 & 26 \\
\hline
\end{tabular}
are further examples which likewise separate the five methods. Of course in many cases, two or more of the methods will agree in their results.

The following table gives a summary of the working rules for the five methods, arranged in the order in which they favor the small states.

It will be observed that the Method of Equal Proportions occupies the central position among the five methods, having no "bias" in favor of either the small or the large states.

- The working rule for Method GD, except for the provision that every state shall have at least one representative, is the same as that devised by the Belgian, Victor d'Hondt, in 1885, as a practical method of computation; none of the theoretical tests $(1,2,3,4 ; 1 a, 2 a, 3 a, 4 a)$ were known at that time. On the history of the d'Bondt Method, see C. G. Hoag and G. H. Hallett, Proportional Representation, New York, 1926. 


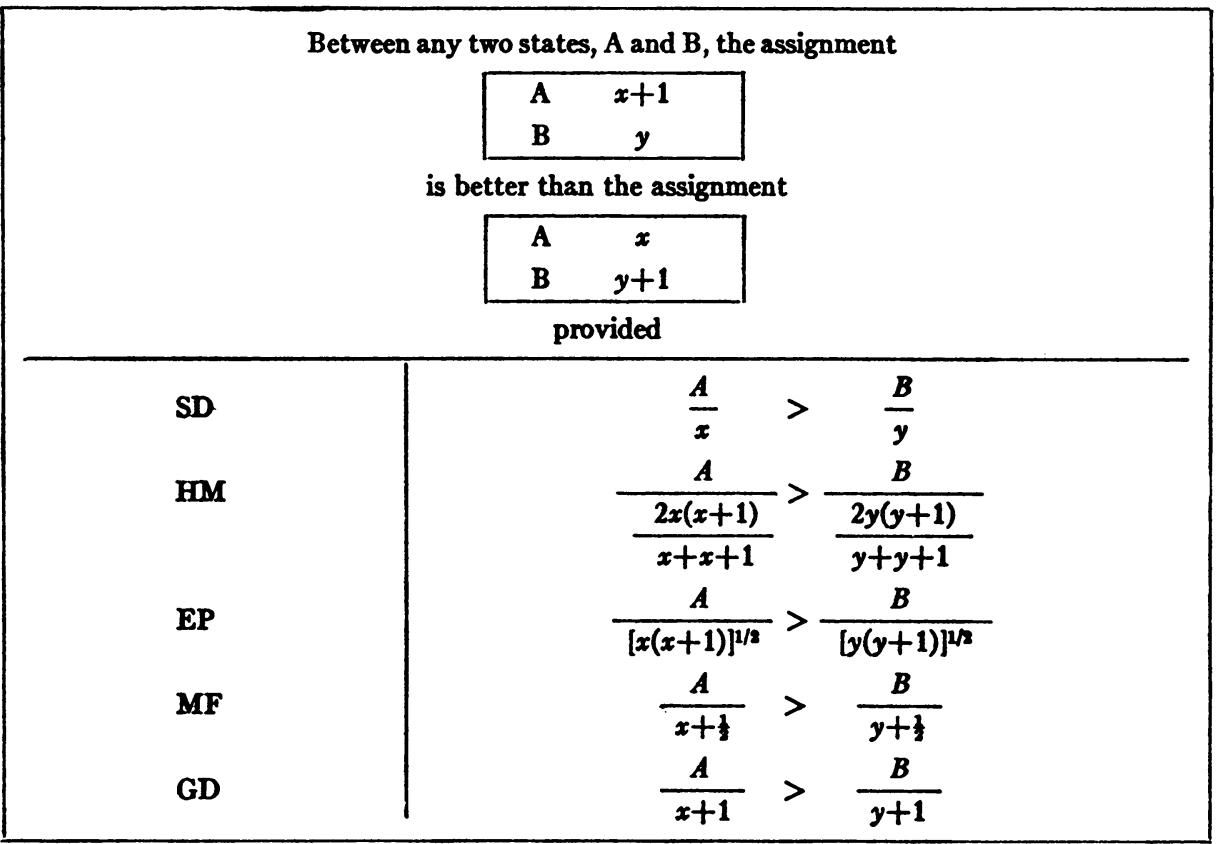

APPENDIX II

Critique of certain unworkable tests

A fifth form in which the exact equation may be written is the following (using the notation explained above):

$$
\frac{\text { rep. over }}{\text { rep. under }}=\frac{\text { Pop. over }}{\text { Pop. under }} \text {, }
$$

and the (relative or absolute) difference between these two numbers might be taken as the measure of inequality between the two states.

If we use the relative difference, the resulting Test 5 (which the reader may write out for himself) leads at once to the Method of Equal Proportions.

If, on the other hand, we use the absolute difference, we have a Test 5a which is not merely less desirable but is absolutely "unworkable."

TEST 5a (unworkable). If the value of the difference

$$
\text { (rep. over)/(rep. under) - (Pop. over)/(Pop. under) }
$$

belonging to any two states can be reduced by a transfer of representatives from one state to the other, then this transfer should be made.

In many cases this apparently plausible test fails to give any information as to which of several proposed apportionments is to be preferred. For example, 
if we attempt to apply this test to the apportionment of 16 representatives to the three states whose populations are given in Example 13, we find that assignment (1) is better than assignment (2), and that (2) is better than (3), and also that (3) is better than (1), so that no choice is indicated.

Examples 14, 15, 16, 17, 18 establish in like manner the unworkableness of certain other tests, which will be listed below.

All these results confirm again our belief that the use of absolute differences, instead of the more natural relative differences, in this problem, is not well advised.

\begin{tabular}{|c|c|c|c|c|c|c|c|c|c|}
\hline \multirow{2}{*}{ Ex. 13} & \multirow{2}{*}{\multicolumn{3}{|c|}{$\begin{array}{c}\text { Assignment of } \\
\text { Reps. }\end{array}$}} & \multicolumn{6}{|c|}{ (rep. over) $/($ rep. under) $-($ Pop. over $) /($ Pop. under) } \\
\hline & & & & $4 / 5-C / B$ & $6 / 3-B / C$ & $6 / 7-B / A$ & $8 / 5-A / B$ & $8 / 3-A / C$ & $4 / 7-C / A$ \\
\hline Pop. & (1) & (2) & (3) & (1) & (2) & (2) & (3) & (3) & (1) \\
\hline $\begin{array}{l}762 \\
534 \\
304\end{array}$ & $\begin{array}{l}7 \\
5 \\
4\end{array}$ & $\begin{array}{l}7 \\
6 \\
3\end{array}$ & $\begin{array}{l}8 \\
5 \\
3\end{array}$ & $\begin{array}{l}0.800 \\
0.569\end{array}$ & $\begin{array}{l}2.000 \\
1.757\end{array}$ & $\begin{array}{l}0.857 \\
0.701\end{array}$ & $\begin{array}{l}1.600 \\
1.427\end{array}$ & $\begin{array}{l}2.667 \\
2.507\end{array}$ & $\begin{array}{l}0.571 \\
0.399\end{array}$ \\
\hline 1600 & 16 & 16 & 16 & 0.231 & 0.243 & 0.156 & 0.173 & 0.160 & 0.172 \\
\hline
\end{tabular}

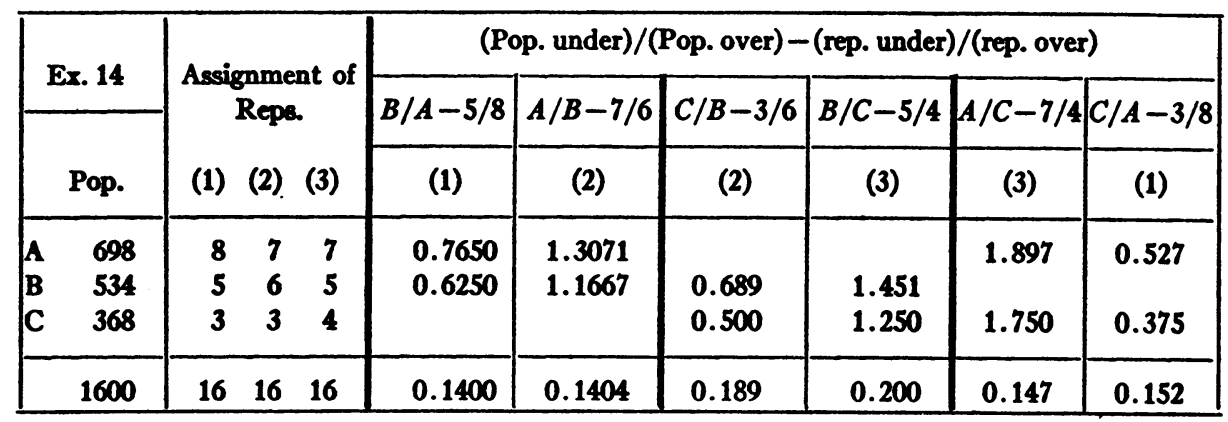

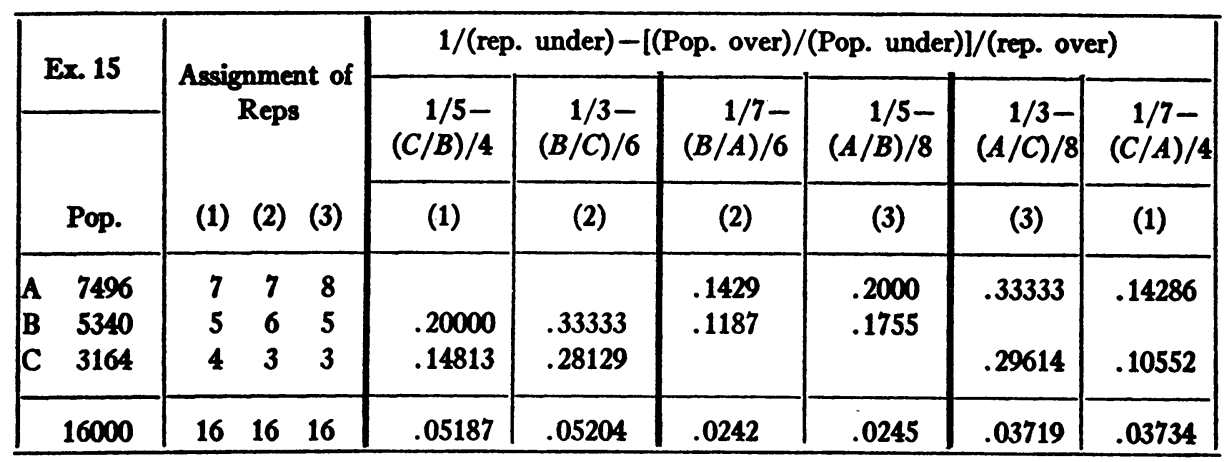




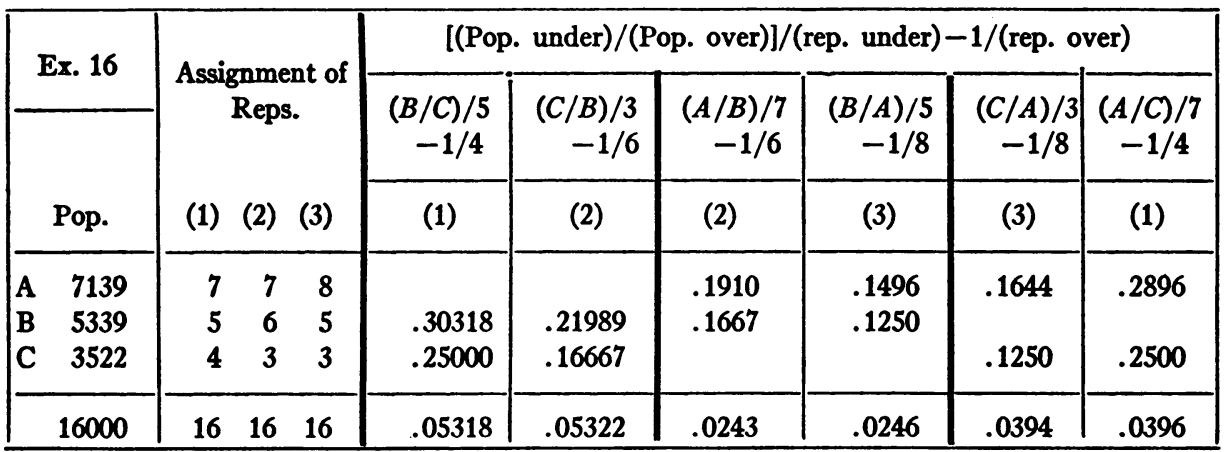

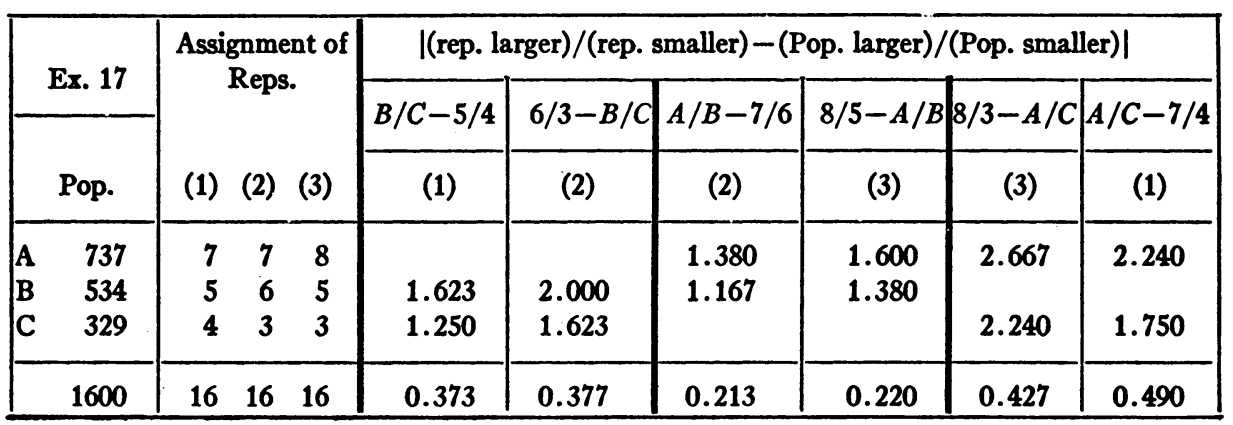

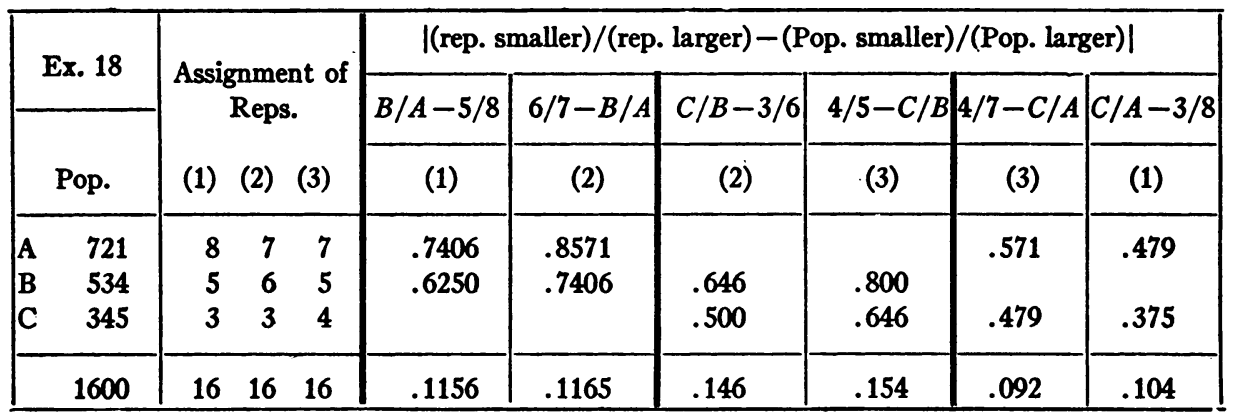

\section{Summary of Tests 1-32 and Tests 1a-32a}

A systematic examination of all the ways in which the exact equation may be written suggests a total of 64 measures of inequality between two states (32 based on the use of relative differences, and 32 based on the use of absolute differences; see table below).

The 32 tests based on relative differences may be called Tests 1-32, and 
all lead to the same Method of Equal Proportions.* The 32 less desirable tests based on absolute differences may be called Tests 1a-32a, and lead to a confusion of miscellaneous results, as exhibited in the accompanying table.

\begin{tabular}{|c|c|c|c|c|c|}
\hline \multicolumn{6}{|c|}{ UNDESIRABLE MEASURES OF INEQUALITY BETWEEN TWO STATES } \\
\hline \multicolumn{3}{|c|}{$\begin{array}{c}\text { In Tests 1a-16a, } \\
A=\text { Pop. of over-represented state } \\
B=\text { Pop. of under-represented state }\end{array}$} & \multicolumn{3}{|c|}{$\begin{array}{l}\text { In Tests 17a-32a, } \\
A=\text { Pop. of larger state } \\
B=\text { Pop. of smaller state }\end{array}$} \\
\hline (1a) & $\frac{B}{b}-\frac{A}{a}$ & HM & (17a) & $\left|\frac{B}{b}-\frac{A}{a}\right|$ & HM \\
\hline (2a) & $\frac{a}{A}-\frac{b}{B}$ & MF & (18a) & $\left|\frac{a}{A}-\frac{b}{B}\right|$ & MF \\
\hline (3a) & $a-\frac{A}{B} b$ & SD & (19a) & $\left|a-\frac{A}{B} b\right|$ & MF \\
\hline (4a) & $\frac{B}{A} a-b$ & GD & (20a) & $\left|\frac{B}{A} a-b\right|$ & MF \\
\hline (5a) & $\frac{a}{b}-\frac{A}{B}$ & Ex. 13 & (21a) & $\left|\frac{a}{b}-\frac{A}{B}\right|$ & Ex. 17 \\
\hline (6a) & $B \frac{a}{b}-A$ & SD & (22a) & $\left|B \frac{a}{b}-A\right|$ & Ex. 17 \\
\hline (7a) & $\frac{1}{A} \frac{a}{b}-\frac{1}{B}$ & SD & (23a) & $\left|\frac{1}{A} \frac{a}{b}-\frac{1}{B}\right|$ & Ex. 17 \\
\hline (8a) & $\frac{B}{A} \frac{a}{b}-1$ & EP & (24a) & $\left|\frac{B}{A} \frac{a}{b}-1\right|$ & Ex. 17 \\
\hline (9a) & $1-\frac{A}{B} \frac{b}{a}$ & EP & (25a) & $\left|1-\frac{A}{B} \frac{b}{a}\right|$ & Ex. 18 \\
\hline (10a) & $B-A \frac{b}{a}$ & GD & (26a) & $\left|B-A \frac{b}{a}\right|$ & Ex. 18 \\
\hline (11a) & $\frac{1}{A}-\frac{1}{B} \frac{b}{a}$ & GD & (27a) & $\left|\frac{1}{A}-\frac{1}{B} \frac{b}{a}\right|$ & Ex. 18 \\
\hline (12a) & $\frac{B}{A}-\frac{b}{a}$ & Ex. 14 & (28a) & $\left|\frac{B}{A}-\frac{b}{a}\right|$ & Ex. 18 \\
\hline (13a) & $\frac{1}{b}-\frac{A}{B} \frac{1}{a}$ & Ex. 15 & (29a) & $\left|\frac{1}{b}-\frac{A}{B} \frac{1}{a}\right|$ & $\mathbf{H M}$ \\
\hline (14a) & $\frac{B}{A} \frac{1}{b}-\frac{1}{a}$ & Ex. 16 & (30a) & $\left|\frac{B}{A} \frac{1}{b}-\frac{1}{a}\right|$ & $\mathbf{H M}$ \\
\hline (15a) & $B a-A b$ & MF & (31a) & $|B a-A b|$ & MF \\
\hline (16a) & $\frac{1}{A b}-\frac{1}{B a}$ & HM & (32a) & $\left|\frac{1}{A b}-\frac{1}{B a}\right|$ & $\mathbf{H M}$ \\
\hline
\end{tabular}

Note. $(17 \mathrm{a})=(1 \mathrm{a}),(18 \mathrm{a})=(2 \mathrm{a}),(31 \mathrm{a})=(15 \mathrm{a}),(32 \mathrm{a})=(16 \mathrm{a})$.

* Tests 1-32 may be read immediately from the Table of Tests 1a-32a by dividing each difference by the smaller of its two terms. The resulting relative difference will be equal to $(a / b) /(A / B)-1$ or $1-(A / B) /(a / b)$, according as $A$ or $B$ is the over-represented state. 
In this table, in Tests 1a-16a, A stands for the over-represented state, and $B$ for the under-represented state; and in Tests 17a-32a, A stands for the larger state, and $B$ for the smaller, while the vertical bars indicate that the absolute value of the quantity is to be taken, without regard to sign. Tests followed by "Ex. 13," “Ex. 14," etc., are "unworkable" tests, the proof of this fact being supplied in each case by the example cited.

It may be noted that measures $17 \mathrm{a}$ and $18 \mathrm{a}$ are the same as measures $1 \mathrm{a}$ and $2 a$, respectively, while measures $31 \mathrm{a}$ and $32 \mathrm{a}$ are the same as $15 \mathrm{a}$ and $16 \mathrm{a}$. Measures 17a, 29a, 30a, 32a differ only by a constant factor; and the same is true of measures $18 \mathrm{a}, 19 \mathrm{a}, 20 \mathrm{a}, 31 \mathrm{a}$, and of measures 21a, 22a, 23a, 24a, and of measures $25 a, 26 a, 27 a, 28 a$.

\section{A FURTHER BASIS FOR THE METHOD OF EQUAL PROPORTIONS}

It may also be noted that Tests $8 \mathrm{a}$ and $9 \mathrm{a}$, although based on absolute differences, happen to lead to the Method of Equal Proportions. The quantity $(a / b) /(A / B)-1$, which occurs in Test $8 \mathrm{a}$, and the quantity $1-(A / B) /(a / b)$, which occurs in Test 9a, may be called, respectively, the (absolute) ratiosurplus and the (absolute) ratio-deficiency belonging to the two states. If we use the term ratio-discrepancy to mean, at pleasure, the relative or absolute ratio-surplus, or the relative or absolute ratio-deficiency, belonging to two states, then the four tests $8,9,8 \mathrm{a}, 9 \mathrm{a}$ may be combined into a single criterion, as follows:

TEST 33. If the "ratio-discrepancy" belonging to any two states (that is, the relative or absolute amount by which $(a / b) /(A / B)$ or $(A / B) /(a / b)$ differs from unity) can be reduced by a transfer of a representative from one state to the other, then this transfer should be made.

This Test 33, like all the Tests 1-32, leads directly to the Method of Equal Proportions. The original Tests 1 and 2 remain, however, perhaps the most satisfactory characterization of the Method.

Briefly, the Method of Equal Proportions may be described as the only method which makes (1) the ratio of population to representatives and (2) the ratio of representatives to population, as nearly uniform as possible among the the several states.

\section{APpendix III}

\section{CRITIQUe OF METHODS BASED ON AVERAGE OR TOTAL ERROR}

All the discussion up to this point has been based on the idea of comparison between competing states, and all the tests so far considered may be called 
"comparison tests." There is another possible method of approach to the problem, however, which should here be mentioned. This is based on the idea of computing some sort of average or total error for the whole apportionment, and selecting as the best apportionment that one whose total error is the least.

There are two objections to this method of approach. In the first place, it is obvious that a total or average error might be reasonably small, while at the same time the error affecting some particular state might be shockingly large; and a gross injustice done to a particular state could hardly be successfully defended on the ground that "on the average" the other states are fairly treated.

In the second place, when one actually tries to set up a definition for the total or average error, the multiplicity of possible formulas makes it extremely difficult to select any one as more significant than the rest. If $q$ is the true quota, and $r$ the actual number of representatives, of the $i$ th state, then the error attached to that particular state may be defined in at least four different ways: $r-q, r / q-1,1-q / r, 1 / q-1 / r$; and the total error may then be defined as the simple sum or as the weighted sum, of either the absolute values of the errors, or the squares of the errors; and the weighting factors may be chosen in a great variety of ways. Most of the resulting methods can be shown to involve the Alabama Paradox; the only ones which do not, lead about equally to the Method of Major Fractions and the Method of Equal Proportions.

Thus, Method MF minimizes

$$
\sum\left[q\left(\frac{r}{q}-1\right)^{2}\right]=\sum\left[r\left(\frac{r-q}{(r q)^{1 / 2}}\right)^{2}\right]=\sum\left[\frac{(r-q)^{2}}{q}\right]
$$

while Method EP minimizes

$$
\sum\left[r\left(1-\frac{q}{r}\right)^{2}\right]=\sum\left[q\left(\frac{r-q}{(r q)^{1 / 2}}\right)^{2}\right]=\sum\left[\frac{(r-q)^{2}}{r}\right] .
$$

As neither of these sets of formulas appears to have any obvious advantages over the other, it is difficult to make out a clear case for either the Method MF or the Method EP on the basis of the idea of total error.*

Finally, it may occur to one to use, as the measure of error of the whole apportionment, not the sum of the errors of the several states, but the maximum error with which any state is affected; the best apportionment being

- See F. W. Owens, loc. cit., and E. V. Huntington, loc. cit. 
that one which has the smallest maximum error. As far as is known, all attempts to apply this principle lead to the Alabama Paradox.

We are thrown back, therefore, on the simple comparison tests, the study of which reveals the substantial advantages of the Method of Equal Proportions.

HARVARD UNIVERSITY, Cambrimge, Mass. 